\title{
Soil Moisture Content Measurement Using Optical Fiber Long Period Gratings
}

\author{
J.S.Hallett ${ }^{\mathrm{a}}$, M.Partridge ${ }^{\mathrm{a}}$, S.W. James ${ }^{* a}$, D. Tiwari ${ }^{\mathrm{a}}$, T. Farewell ${ }^{\mathrm{b}}$, S.H. Hallett ${ }^{\mathrm{b}}$, and R.P.Tatam ${ }^{\mathrm{a}}$ \\ ${ }^{\mathrm{a}}$ Engineering Photonics, ${ }^{\mathrm{b}}$ Cranfield Soil and Agrifood Institute, Cranfield University, \\ Bedfordshire, MK43 0AL, UK
}

\begin{abstract}
The use of an optical fibre long period grating (LPG) as a soil moisture sensor is reported. Characterization of the device in both clay and sandy soils revealed a sensitivity to moisture levels in the range 10-50\%, and the results were compared with the output from a Theta probe, the standard soil moisture sensor, which measures the impedance of the soil.
\end{abstract}

Keywords: Long Period Grating, Fiber optic, gratings, soil, moisture.

\section{INTRODUCTION}

The measurement of soil moisture content is essential in agriculture and for the planning and management of buried assets ${ }^{1}$. Currently available soil moisture measurement devices are restricted to observations recorded at discrete locations ${ }^{2}$. There is a need for new technology to facilitate the development of a holistic approach, able to analyse a large area concurrently. For example, this might include the detection of leakages along a water pipeline network, or in applications such as agricultural field management and agronomic irrigation scheduling. Optical fiber sensors have the potential to address this; providing a relatively inexpensive solution which can detect water over both multiple locations and great distances. Such a device should be capable of detecting a range of moisture levels in a variety of soil types and ground conditions.

The sensitivity of an optical fiber long period grating (LPG) to the refractive index of its surrounding medium is welldocumented $^{3}$. This has been used to demonstrate refractometers for measuring the concentration of solutions or the curing of resins ${ }^{4}$, chemical sensors by exploiting coatings that change their refractive indices in response to an analyte ${ }^{5}$, and liquid level sensors ${ }^{6}$. Here, the refractive index sensitivity of LPGs is exploited to measure the moisture content of soil.

Two contrasting soil types were selected for the experimental investigation of the sensor. One was an agricultural soil from the 'Wickham' soil series ${ }^{7}$, being a typic stagnogley, comprising medium silty drift over clayey material passing to clay, and being imperfectly drained with pore spacing of $<2 \mu \mathrm{m}$. The other sample was a loamy sand horticultural organic soil, derived on the 'Cottenham' soil series ${ }^{7}$, being a typic brown sand, comprising ferruginous medium to coarse sandy material passing to a parent material of Lower Greensand, and being freely draining with pore spacing in range 60$2000 \mu \mathrm{m}$. These were selected to offer contrasting soil moisture retention and mineral pore space characteristics, as well as being typical of soils in anticipated application environments. This permitted the performance of the sensor to be assessed in a range of soil physical, textural and hydrological conditions. A Theta Probe (SM200) was used as a reference instrument for determining volumetric moisture content. The probe operates by measuring the electrical resistivity between two terminals inserted into the soil, which is related to the water content in the body of soil between the rods. The quoted accuracy of the device is $\pm 3 \%{ }^{8}$. Volumetric water content, or moisture content, $\theta v$, is widely used to quantify soil moisture ${ }^{2}$. It is expressed as a ratio of water volume to total volume $\left(\mathrm{m}^{3} \cdot \mathrm{m}^{-3}\right)$ :

$$
\theta v=\text { VolWater/VolSample }
$$

*s.w.james@cranfield.ac.uk; phone +44 (0) 1234 754623; openoptics.info

\footnotetext{
25th International Conference on Optical Fiber Sensors, edited by Youngjoo Chung, Wei Jin,

Byoungho Lee, John Canning, Kentaro Nakamura, Libo Yuan, Proc. of SPIE Vol. 10323,

103232J · () 2017 SPIE · CCC code: 0277-786X/17/\$18 · doi: 10.1117/12.2263427

Please refer to any applicable publisher terms of use.

Proc. of SPIE Vol. 10323 103232J-1
} 
where VolWater is the volume of water in the soil sample, and VolSample is the total volume of dry soil, air and the water in the sample. It is commonly converted to a percentage for a more intuitive representation. This investigation tested soil to its field capacity, a term referring to the amount of soil moisture or water content held in the soil after excess water has drained away.

\section{MATERIALS \& METHODS}

\subsection{LPG Fabrication}

The LPG, of period $109 \mu \mathrm{m}$ and length $40 \mathrm{~mm}$, was fabricated in a boron-germanium, co-doped photosensitive optical fiber, with a cut-off wavelength of $670 \mathrm{~nm}$ (Fibercore PM750) by point-by-point UV irradiation using the overwrite method $^{9}$. The period of the LPG was selected such that one of the resonance bands, centered at $850 \mathrm{~nm}$ was at the phase matching turning point (PMTP). The transmission spectrum of the optical fiber was recorded by coupling the output from a tungsten-halogen lamp (Ocean Optics LS-1) into the fiber, analyzing the transmitted light using a fiber-coupled CCD spectrometer (Ocean Optics S2000).

\subsection{Sensor packaging}

The packaging used in deploying the LPG has to mitigate the risks to the integrity of the fibre posed by contact with abrasive soil minerals, whilst maintaining optimal performance. A 3D printed structure for packaging the LPG was developed in order to protect the fibre from damage while also allowing soil water to flow freely around it. The packaging developed is shown in Figure 1.

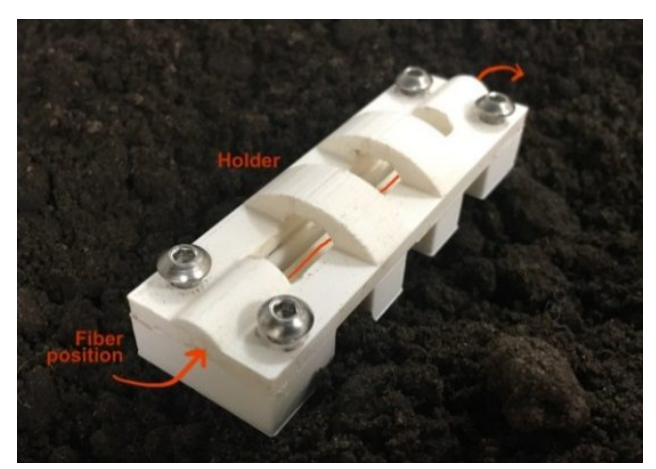

Figure 1: Photograph of the 3D printed fiber holder. The housing has dimensions $80 \mathrm{~mm} \times 25 \mathrm{~mm} \times 10 \mathrm{~mm}$

The LPG was clamped within the casing, designed to protect the uncoated fiber and to keep it taut to avoid the influence of flexing. Arches were placed over the casing to trap soil within whilst still permitting moisture to pass through. The design was such that, when the sensor was moved, the soil sample was restricted physically inside the casing to prevent it moving across the LPG, thus avoiding abrasion or torsion to act on the fiber. Free drainage is also permitted via the slotted underside design of the package, avoiding water accumulation.

\subsection{Moisture Measurements}

Moisture measurements were conducted in two different types of soil: clay and sandy loam. The soil sample in the experiment did not have its 'pore space' calculated (bulk density), as the experiment involved by design the repeated wetting/drying of the sample - so removing natural structure. It was viewed that this approach was pragmatic for the 'proof of concept', and furthermore, two contrasting soils were selected, one having naturally large pore spaces. A future experiment would involve emplacing the probe in a field condition and after equilibrium and settling taking more natural readings.

Alongside the LPG, a commercial water sensor, the Theta Probe (SM200), was used to measure the soil moisture content for comparison. As temperature affects the spectral output of the fiber ${ }^{3}$, a thermocouple, connected to a data logger (Pico Logger), was used to monitor the temperature. The soil was placed in a container of dimensions 300x200x100 mm, and the LPG was buried to a depth of $50 \mathrm{~mm}$, with the Theta probe and thermocouple placed in close proximity at the same depth, ensuring that the fiber and the orientation plane in which the Theta probe measured were held parallel to the 
bottom of the container. The sensors were allowed to acclimatize to the ambient soil moisture. Water was then added by syringe uniformly to an area of $120 \times 80 \mathrm{~mm}$ on the soil's surface. The addition of water was progressive, at a rate of approximately $10 \mathrm{ml}$ of water being added every $30 \mathrm{~s}$. Water was added to the dry soil samples until the theta probe measurements stabilized, whereby excess water drained off. For sandy soil this equated to around $300 \mathrm{ml}$ and for clay $500 \mathrm{ml}$. This is equivalent to the critical agronomic state of 'soil field capacity'. The results recorded comprised spectra from the LPG, soil moisture values from the theta probe, and a log of soil temperature from the thermocouple, as well as time and water added.

\section{RESULTS \& DISCUSSION}

In order to demonstrate the sensitivity of the LPG, irrespective of the addition of soil, the LPG was initially immersed in deionized water. The spectrum was recorded before and after immersion to show the spectral change (Figure 2).

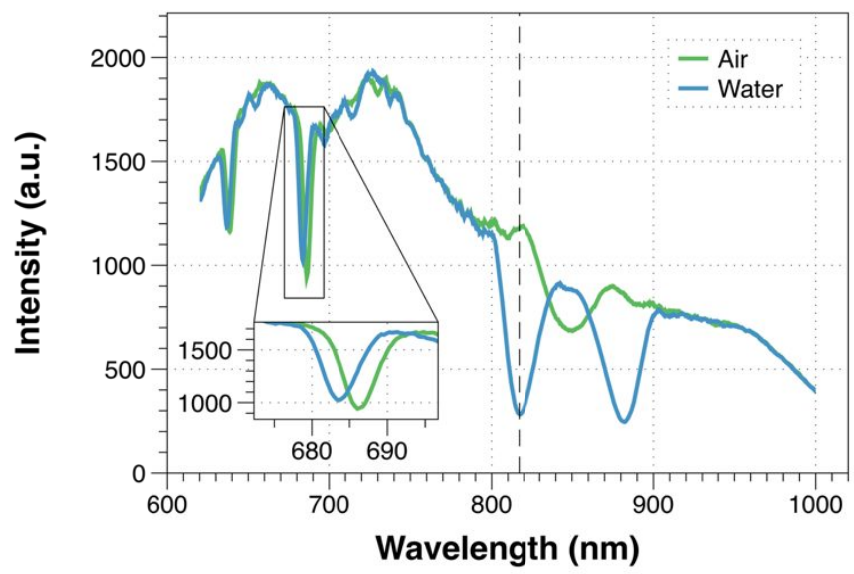

Figure 2: Transmission spectra of the LPG when in air and in water. The expanded section highlights the resonance band corresponding to coupling $\mathrm{LP}_{018}$, and the dotted line denotes $817 \mathrm{~nm}$.

Figure 2 reveals a distinctive shift in the LPG when immersed in water. This shift is characteristic of a surrounding refractive index increase from 1.0 to 1.33 . The resonance band corresponding to coupling to $\mathrm{LP}_{0}{ }_{19}$ (between 800 and 900 $\mathrm{nm}$ ) shifts from the edge of the PMTP to the point where the dual resonance bands have formed. The resonance band corresponding to coupling to $\mathrm{LP}_{018}$ is shown in detail in the inset in Figure 2, showing a shift but, as it is further away from the PMTP, the shift is smaller in magnitude $(\sim 3 \mathrm{~nm})$.

Typically, LPG sensors are monitored by tracking the centre wavelengths of the resonance bands. However, for this grating period, an increase in the surrounding refractive index causes an initial further deepening of the resonance band at the PMTP. However, monitoring the transmission at a single wavelength $(817 \mathrm{~nm})$ provides a monotonically decreasing transmission in response to increasing refractive index. Figures 3 (a) and 3 (b) show the results from the Theta probe and from the LPG sensor in clay and sand samples, respectively.

In both soil types, the LPG sensor shows correlation with the readings from the Theta probe, but there is a time delay of approximately 3 minutes between the two sensors systems (Figure 3). Additionally, the magnitude of the change in the LPG sensor appears to be non-linear when compared to the Theta probe. This non-linearity arises due to the design of the LPG sensor, as working near the PMTP gives high sensitivity but poor linearity. This non-linearity of response is expected and when used practically can be mitigated with the use of calibration and lookup curves.

The time delay in the LPG results is likely a result of the combination of positional differences in the LPG and theta probe and the housing of the LPG. While the prototype housing kept the fiber secure, it was also restrictive and could have caused a delay in the time taken for water to infiltrate the device. The prongs of the theta probe had no such housing issues, and so water is able to move freely between them. The housing used in this work was the first prototype, future prototypes will be modified to allow for freer movement of soil and water around the sensing region. 

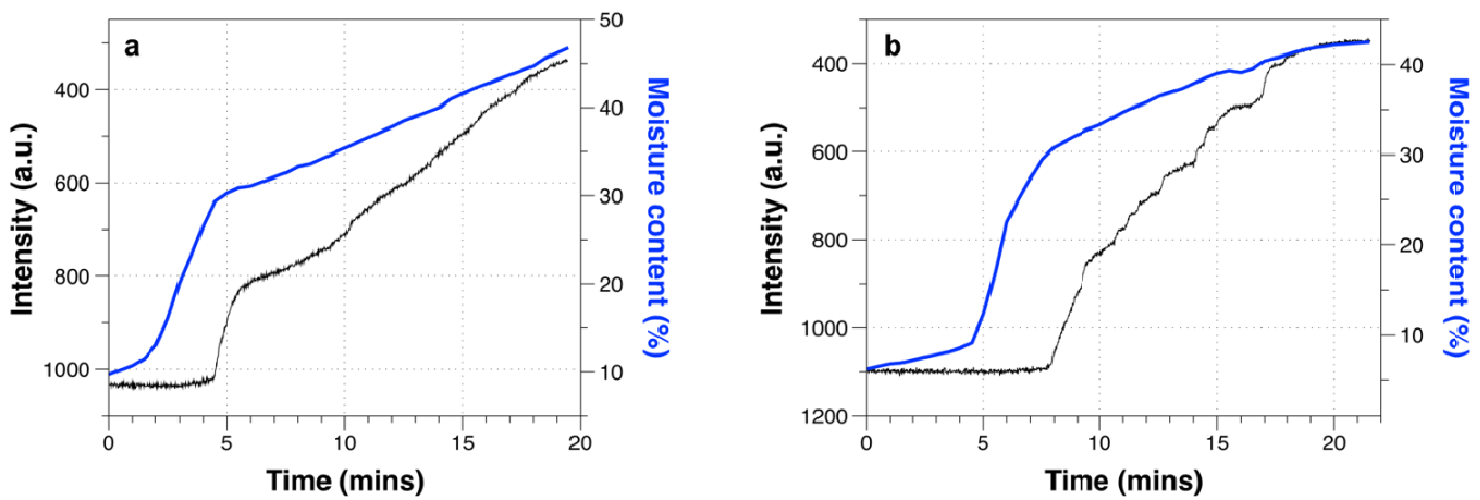

Figure 3: Comparison of the intensity at $817 \mathrm{~nm}$ of transmission through the LPG sensor (in black) to the moisture content as measured by Theta probe (blue) for sandy (a) and clay (b) soil.

\section{CONCLUSIONS}

The performance of a prototype LPG-based moisture sensor, characterized within both sandy loam and clay soil environments has been reported, showing sensitivity to a range of moisture concentrations from 10 to $50 \%$. While this work is at an early stage, this demonstrates a real world practical application of a simple un-coated LPG based moisture sensor. Future work with this sensor system will be the deployment of this sensor in external testing sites near water pipelines and at agricultural sites.

\section{ACKNOWLEDGEMENTS}

JH would like to acknowledge the support of The Natural Environment Research Council (NERC) (NE/M009009/1).

The underlying data can be found at http://dx.doi.org/10.17862/cranfield.rd.3969291 and http://dx.doi.org/10.17862/cranfield.rd.3969354.

\section{REFERENCES}

[1] Lekshmi, S. U., Singh, D. N., Shojaei Baghini, M., "A critical review of soil moisture measurement," Measurement 54, 92-105 (2014).

[2] Paige, G. B., Keefer, T. O., "Comparison of Field Performance of Multiple Soil Moisture Sensors in a Semi-Arid Rangeland," J. Am. Water Resour. Assoc. 44(1), 121-135 (2008).

[3] James, S. W., Tatam, R. P., "Optical fibre long-period grating sensors: characteristics and application," Meas Sci Technol 14(5), 49-62 (2003).

[4] Buggy, S. J., Chehura, E., James, S. W., Tatam, R. P., "Optical fibre grating refractometers for resin cure monitoring," J. Opt. A: Pure App. Opt., 9, 60-65 (2007)

[5] Partridge, M., Wong, R. Y. N., James, S. W., Davis, F., Higson, S. P. J., Tatam, R. P., "Long period grating based toluene sensor for use with water contamination," Sensor. Actuat. B-Chem. 203, 621-625, Elsevier B.V. (2014).

[6] Fu, H., Shu, X., Zhang, A., Liu, W., Zhang, L., He, S., Bennion, I., "Implementation and Characterization of Liquid-Level Sensor Based on a Long-Period Fiber Grating Mach Zehnder Interferometer,” IEEE Sensors Journal 11(11), 2878-2882 (2011).

[7] Canfield University "LandIS," 2016 < http://www.landis.org.uk > (29 October 2016)

[8] Webb, N., "User Manual for the HH2," Delta-T Devices Ltd 1-124 (2013).

[9] Partridge, M., James, S. W., Barrington, J., Tatam, R. P., "Overwrite fabrication and tuning of long period gratings," Opt. Express 24(20), 22345-12 (2016). 
2017-04-23

\section{Soil moisture content measurement using optical fiber long period gratings}

Hallett, Stephen $\mathrm{H}$.

SPIE - International Society for Optics and Photonics

J.S. Hallett, M. Partridge, S.W. James, D. Tiwari, T. Farewell, S.H. Hallett, R.P. Tatam. Soil moisture content measurement using optical fiber long period gratings. 25th International Conference on Optical Fiber Sensors, 24-28 April 2017, Jeju, Republic of Korea http://dx.doi.org/10.1117/12.2263427

Downloaded from Cranfield Library Services E-Repository 